\title{
Effect of the Grafting Reaction of Aluminum Nitride on the Multi-Walled Carbon Nanotubes on the Thermal Properties of the Poly(phenylene sulfide) Composites
}

\author{
Myounguk Kim ${ }^{1,2}$, Sunmin Park ${ }^{2, *}$ and Jongshin Park ${ }^{1, *}$ \\ 1 Department of Biosystems \& Biomaterials Science and Engineering, Seoul National University, Seoul 08826, \\ Korea; myounguk@snu.ac.kr \\ 2 Ceramic Fiber \& Composite Center, Korea Institute of Ceramic Engineering and Technology, \\ Jinju 52851, Korea \\ * Correspondence: psm@kicet.re.kr (S.P.); jongshin@snu.ac.kr (J.P.); \\ Tel.: +82-55-792-2624 (S.P.); +82-2-880-4628 (J.P.)
}

Received: 8 August 2017; Accepted: 13 September 2017; Published: 15 September 2017

\begin{abstract}
In this study, the PPS/MWCNTs/AIN composite was prepared with poly(phenylene sulfide) (PPS), covalent functionalized multi-walled carbon nanotubes (fMWCNTs), and aluminum nitride (AlN) via melt-blending techniques. The AlN is a fascinating non-oxidizing ceramic material having the highest thermal conductivity among the ceramic materials. In order to introduce the functional groups on the surface of the AlN particles, a silane coupling agent was used as it is able to graft with the functional groups on the covalent functionalized MWCNTs. The silanization reaction of the AlN was confirmed qualitatively and quantitatively by FT-IR (Fourier Transform Infrared Spectroscopy), and XPS (X-ray Photoelectron Spectroscopy). The grafting reaction of the AlN particles on the MWCNTs was confirmed using UV-Vis (Ultraviolet-Visible Spectroscopy), FE-SEM (Field-Emission Scanning Electron Microscopy) and FE-TEM (Field-Emission Transmission Electron Microscopy) images. The grafting reaction was accomplished by observing the change of the transmittance, the morphology of the AlN particle bonded to the MWCNTs. For the morphological changes of the fractured surface of the PPS/MWCNTs/AIN composites by FE-SEM, the hybrid filler was homogeneously dispersed on the PPS matrix when the AlN particle was grafted on the MWCNTs. The homogeneous distribution of the hybrid filler acts as a heat transfer path, which led the higher thermal properties, such as thermal conductivity, thermal resistance, and melting temperature than those of not grafted MWCNTs.
\end{abstract}

Keywords: poly(phenylene sulfide); multi-walled carbon nanotubes; aluminum nitride; grafting reaction; thermal property

\section{Introduction}

Recently, the uses of the super-engineering plastic have steadily increased for various industrial applications due to their higher heat and chemical resistance and physical properties than the commodity polymers have been used. In this study, poly(phenylene sulfide) (PPS), which is one of the super-engineering plastics, was used as a thermal conductive polymer matrix of the composite. The PPS is known for a semi-crystalline aromatic thermoplastic polymer possessing a high melting point $\left(T_{m}\right)$, at approximately $285^{\circ} \mathrm{C}$, excellent mechanical properties, thermal resistance, and excellent flame and chemical resistance. Despite its high melting temperature, the PPS has limited applications due to it having a low glass temperature $\left(T_{g}\right)$ by flexible sulfide linkages in their own chemical 
structures and brittleness. In order to overcome these limitations, blending the PPS matrix with other organic/inorganic compounds has been used for preparing the composites [1,2].

Since carbon nanotubes (CNTs) with tubular structure of a graphene sheet were firstly reported twenty-five years ago by lijima et al. [3], The CNTs have been used as a conducting filler to introduce the thermal and electrical conductivity on non-conductive polymers because of their excellent mechanical properties, thermal and electrical properties and having a high aspect ratio [4-7]. Among various properties of the polymer nanocomposites, thermal conductivity and heat resistance are significant properties applying the polymer nanocomposites to the automotive industry. In order to be used as a thermal resistant material for automobiles, the thermal conductivity should approximately reach 1 to $30 \mathrm{~W} / \mathrm{mK}$ [8]. The heat resistant material may be a composite material comprising a thermally conductive filler such as ceramic and carbon material. Therefore, several researches have been conducted to improve the thermal properties of various polymers using CNTs as thermal conductive fillers. Kostagiannakopoulou et al. and Ciecierska et al. conducted a study to improve the thermal conductivity from $0.29 \mathrm{~W} / \mathrm{mK}$ for neat epoxy to $0.36 \mathrm{~W} / \mathrm{mK}$ for $3 \mathrm{wt} \%$ MWCNTs loaded epoxy composites and $0.13 \mathrm{~W} / \mathrm{mK}$ for neat epoxy to 0.24 for $5 \mathrm{wt} \%$ MWCNTs loaded epoxy composites $[9,10]$. Deng et al. conducted the study to improve thermal conductivity and crystallization properties by introducing carbon based filler such as MWCNTs, and carbon fiber on the PPS matrix [11]. However, the covalent functionalization was widely used for modifying the surface of the MWCNTs because the MWCNTs can be easily aggregated with themselves, which adversely affected the thermal properties of the polymer nanocomposites, due to strong van der Waal's interactions between individual tubes $[12,13]$. The hybrid filler system on the polymer matrix has given rise to a new trend for developing higher thermal conductive composite. The hybrid filler system plays an important role in the nature of the interface and the formation of thermally conductive paths in a composite [14]. Therefore, various ceramic fillers have been used in the hybrid filler system on the polymer matrix for enhancing the thermal conductivity and thermal properties of the composites, such as boron nitride $(\mathrm{BN})$, aluminum nitride $(\mathrm{AlN})$, silicon nitride $\left(\mathrm{Si}_{3} \mathrm{~N}_{4}\right)$ [15-17], aluminum oxide $\left(\mathrm{Al}_{2} \mathrm{O}_{3}\right)$, zinc oxide $(\mathrm{ZnO})$, and beryllium oxide $(\mathrm{BeO})$ [18-20].

In this study, the AlN was additionally introduced by grafting on the MWCNTs on the PPS matrix. The grafting reaction was chosen to one way to form the hybrid filler system for improving thermal properties, such as thermal conductivity, and thermal resistance by distributing uniformly of the fillers on the polymer matrix [21,22]. The AlN was used as ceramic filler to improve a thermal conductivity. The AlN possesses the low intrinsic thermal resistance, the highest thermal conductivity among the ceramic materials, and good modulus [23,24]. Although the AlN has the high intrinsic thermal conductivity among the ceramic materials, the composites having the AlN particle as the filler exhibit the lower thermal conductivity than the expected, because of their aggregations between the particles [25]. In order to prevent these aggregations, the functional group was introduced by chemical treatments capable of a grafting reaction on the MWCNTs, $\gamma$-glycidoxypropyltrimethoxysilane (GPTMS) was used for modifying the surface of the AlN particles.

To the best of our knowledge, the study on the PPS/MWCNTs/AIN composites has not been conducted and reported yet. Therefore, this study aims to describe the effect of the grafting reaction of the AlN particles on the MWCNTs on the distribution on the PPS matrix and thermal properties of the PPS composites.

\section{Experimental}

\subsection{Materials}

The PPS powder used as the polymer matrix of the composite was purchased from Zhejiang NHU Special Materials Co., Ltd., Shaoxing, China. The density of PPS was $1.36 \mathrm{~g} / \mathrm{cm}^{3}$ at $25^{\circ} \mathrm{C}$. The MWCNTs (CM-130) used in this study were synthesized via a catalytic chemical vapor deposition (CVD) process. They were purchased from Hanwha Nanotech Co., Ltd., Daejeon, Korea. The 
MWCNTs purchased consists of 15 multiple walls. The outer diameter ranged from 10 to $15 \mathrm{~nm}$, and the aspect ratio was known as $2 \times 10^{3}$. The purity and density were $>90 \mathrm{wt} \%$ and $0.05 \mathrm{~g} / \mathrm{cm}^{3}$, respectively. Hydrogen peroxide used to functionalize the MWCNTs was purchased from Junsei Chemical Co., Ltd., Tokyo, Japan. The AlN particle having $4 \mu \mathrm{m}$ mean particle size was purchased from Alfa Aesar Chemical Co., Ltd., Incheon, Korea. Its density was known as $3.26 \mathrm{~g} / \mathrm{cm}^{3}$ at $25^{\circ} \mathrm{C}$. $\gamma$-Glycidoxypropyltrimethoxysilane (GPTMS), and ethanol acetone were purchased from Samchun Pure Chemical Co., Ltd., Pyeongtaek, Korea. Tetrahydrofuran (THF), and acetone used to graft AlN particles on the MWCNTs were purchased from Junsei Chemical Co., Ltd., Tokyo, Japan, and Samchun Pure Chemical Co., Ltd., Pyeongtaek, Korea, respectively.

\subsection{Covalent Functionalization of the MWCNTs}

Dried MWCNTs was added into a 1:1 volume ratio solution of $\mathrm{H}_{2} \mathrm{O}_{2} / \mathrm{H}_{2} \mathrm{O}$. The suspension was continuously stirred for $10 \mathrm{~h}$ at $80^{\circ} \mathrm{C}$ to introduce the hydroxyl groups on the surface of the MWCNTs. After stirring, the MWCNTs were filtered and cleaned several times with distilled water until the $\mathrm{pH}$ of the supernatant became neutral, and the filtered MWCNTs powder was dried at $120^{\circ} \mathrm{C}$ in a vacuum oven over-night (covalent functionalized MWCNTs, fMWCNTs) [26].

\subsection{Surface Modification of the AlN Particles}

It is necessary to introduce the functional groups which are capable of the rafting reaction with the functional group of the MWCNTs. Therefore, surface modification of AlN particles was performed using a GPTMS as shown in Figure 1a. The AlN particles, and the GPTMS were added into ethanol and stirred continuously at $80^{\circ} \mathrm{C}$ for $5 \mathrm{~h}$. The suspension was filtered and washed with distilled water to eliminate non-reacted organosilanes. Then, the filtered AIN powder was dried at $120^{\circ} \mathrm{C}$ in a vacuum oven overnight (Silane treated AlN).

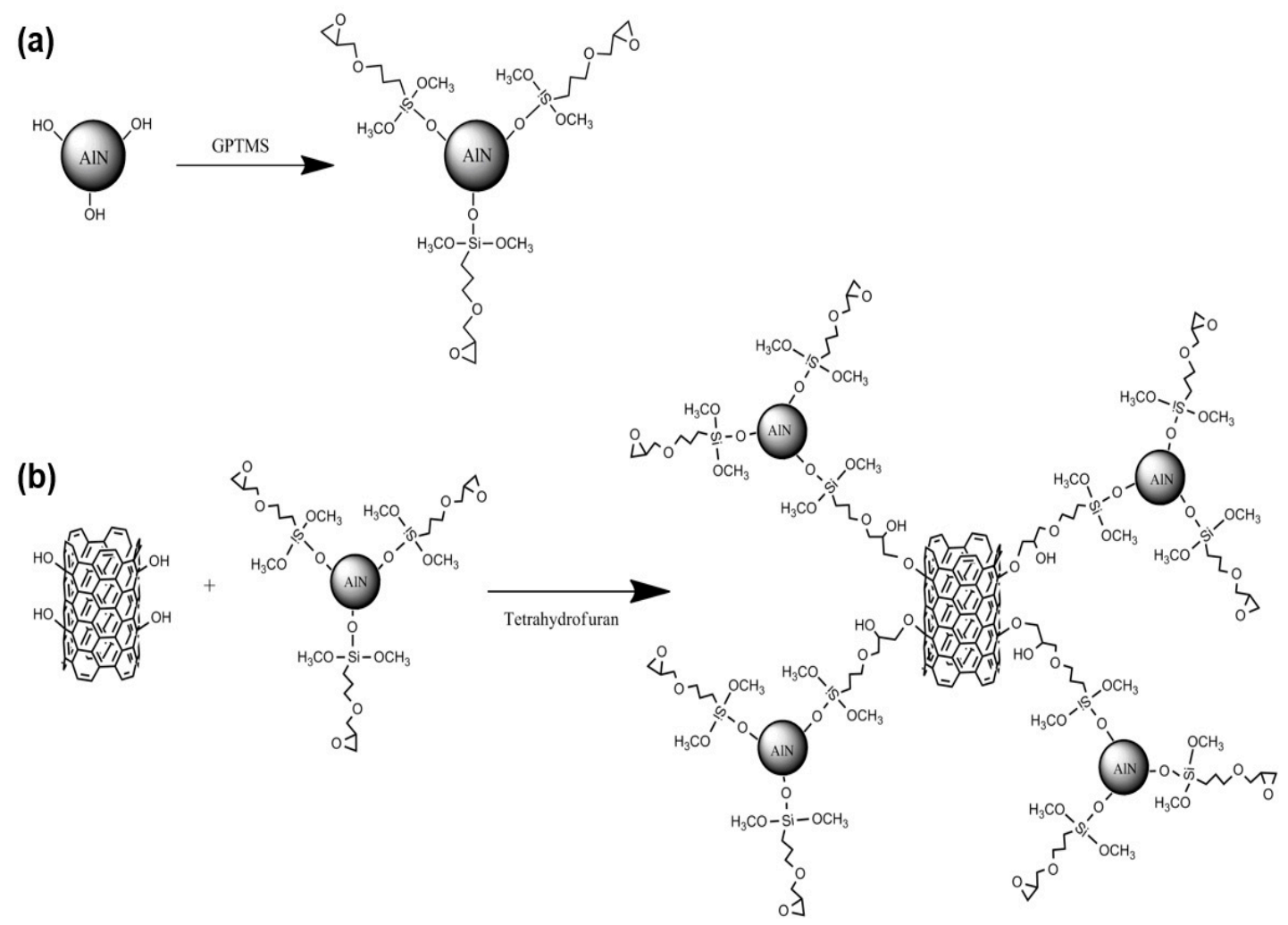

Figure 1. Schemes of the (a) silane treatment of AlN and (b) grafting reaction of the silane treated AlN on the covalently functionalized MWCNTs (fMWCNTs). 


\subsection{The Grafting Reaction of the AlN Particles on the MWCNTs}

The grafting reaction of the AlN particles on the MWCNTs was performed as shown in Figure $1 \mathrm{~b}$. The fMWCNTs and silane treated AlN were added into THF and stirred continuously at $60{ }^{\circ} \mathrm{C}$ for $1 \mathrm{~h}$. The suspension was filtered and washed with acetone to remove the residual solvent. Then, the filtered hybrid filler was dried at $120^{\circ} \mathrm{C}$ in a vacuum oven overnight (AlN grafted MWCNTs).

\subsection{Preparation of the PPS/MWCNTs/AlN Composites}

The PPS/MWCNTs/AIN composite was prepared via a melt-blending technique with various contents of AlN particle. The fMWCNTs, AlN, and PPS powders were poured into absolute ethanol. The mixture was sonicated for $5 \mathrm{~min}$ in a ultra-sonication bath (SD-250H, 290W, Mujigae Co., Seoul, Korea) and then dried in a vacuum oven at $120^{\circ} \mathrm{C}$ until all moisture and solvents evaporated. Then, the PPS/MWCNTs/AlN mixture was melt-blended at $310^{\circ} \mathrm{C}$ using a micro-extruder (Wellzoom, Seoul, Korea) and then compressed using a hot press technique with a two-post manual hydraulic press (\#2699, Carver Inc., Wabash, IN, USA) at $290^{\circ} \mathrm{C}$ under $20 \mathrm{MPa}$. Finally, the mold was cooled in the air to room temperature until the sample hardened. The sample composition of the PPS/MWCNTs/AIN composite was shown in Table 1.

Table 1. The compositions of the PPS/MWCNTs/AlN composites.

\begin{tabular}{cccc}
\hline Sample code & $\begin{array}{c}\text { fMWCNTs } \\
(\mathbf{w t} \%)\end{array}$ & AlN (wt \%) & Silane treated AlN (wt \%) \\
\hline PPSHP1 & & - & - \\
PPSHP1AlN10 & 1 & 10 & - \\
PPSHP1AlN20 & & - & 10 \\
PPSHP1sAlN10 & & & 20 \\
PPSHP1sAlN20 & & & \\
\hline
\end{tabular}

\subsection{Characterization}

A spectrum One Fourier transform infrared spectrophotometer (Perkin Elmer, Shelton, CA, USA) was used in transmission mode to observe the introduced silane coupling agent on the surface of the AlN particles. The obtained spectra ranged from 400 to $4000 \mathrm{~cm}^{-1}$ at a resolution of $4 \mathrm{~cm}^{-1}$. XPS measurement was performed using a monochromatic $\mathrm{Al} \mathrm{K \alpha}$ X-ray source $(15 \mathrm{kV}, \mathrm{h} v=1486.6 \mathrm{eV})$ to confirm the introduction of the silane coupling agents on the surface of AlN particles by observing the $\mathrm{Si}_{2 p}$ peak and $\mathrm{N}_{1 \mathrm{~s}}$ peaks, respectively. Field-Emission Scanning Electron Microscopy (FE-SEM, SU70, Hitachi, Tokyo, Japan), was used to observe the AlN particles grafted on the MWCNTs, and the dispersion differences of the hybrid filler on the PPS matrix depending on the grafting of the AlN on the MWCNTs. Before the FE-SEM characterization, the fractured surface of the composite was sputter coated with a thin layer of platinum. UV-Visible spectrophotometer (UV-Vis, Optizen 2120UV, Mecasys, Daejeon, Korea) was used to confirm that AlN particles was grafted well on the MWCNTs. The suspension was prepared by dispersing $0.2 \mathrm{~g}$ powder (grafted or not) in $25 \mathrm{~mL}$ ethanol. Then, the suspension was continuously stirred for $30 \mathrm{~min}$ at room temperature, and stayed free for 1 day. Field-Emission Transmission Electron Microscopy (FE-TEM, G2 F30 S-TWIN, Tecnai, Eindhoven, Holland) was also used to observe the AlN particles grafted on the MWCNTs. The thermal conductivity of the PPS/MWCNTs/AIN composite was measured using a C-therm TCI thermal conductivity analyzer by the modified transient plane source method (ASTM D7984) at room temperature under normal atmosphere. The measured testing value was averaged five times for each sample. A thermogravimetric analyzer (Q-5000 IR, TA Instruments, Inc., New Castle, DE, USA) was used in order to observe the changes in the thermal resistant property of the PPS/MWCNTs/AIN composites depending on the grafting reaction. The sample was heated from room temperature to $800^{\circ} \mathrm{C}$ at a heating rate of $10^{\circ} \mathrm{C} / \mathrm{min}$ under a nitrogen atmosphere. A differential scanning calorimeter 
(DSC-Q1000, TA Instruments, Inc., New Castle, DE, USA) was used in order to observe the changes in the crystallinity, crystallization temperature, and melting temperatures depending on grafting. The sample was heated to $310^{\circ} \mathrm{C}$ at $10^{\circ} \mathrm{C} / \mathrm{min}$ and held for $5 \mathrm{~min}$ to remove the thermal history within the sample. Then, the sample was cooled to room temperature at $10^{\circ} \mathrm{C} / \mathrm{min}$. The second heating run was performed in the same way with the first heating run.

\section{Results and Discussion}

\subsection{Characterization of the Surface Modification of the AlN Particles}

As mentioned above, surface modification was performed in order to be able to graft of AlN particles on the MWCNTs. To confirm the GPTMS, silane coupling agent was introduced well on the surface of the AlN particles, the FT-IR spectra of untreated AlN particles and silane treated AlN were compared in Figure 2. In the case of the untreated AlN particles, a broad peak only appeared in the spectrum near $720 \mathrm{~cm}^{-1}$ attributed to stretching and bending of the Al-N bond, whereas in the case of the silane treated AlN, additional peaks appeared due to the attached GPTMS. In more detail, two peak appeared at 2977 and $2894 \mathrm{~cm}^{-1}$ attributed to stretching and bending of the methylene groups in the attached GPTMS. Moreover, another peak appeared at $1067 \mathrm{~cm}^{-1}$ attributed to the Si-O stretching in the GPTMS [27]. As a result, it was confirmed that surface modification was performed well by observing the several peaks of the FT-IR spectrum of the silane treated AlN.

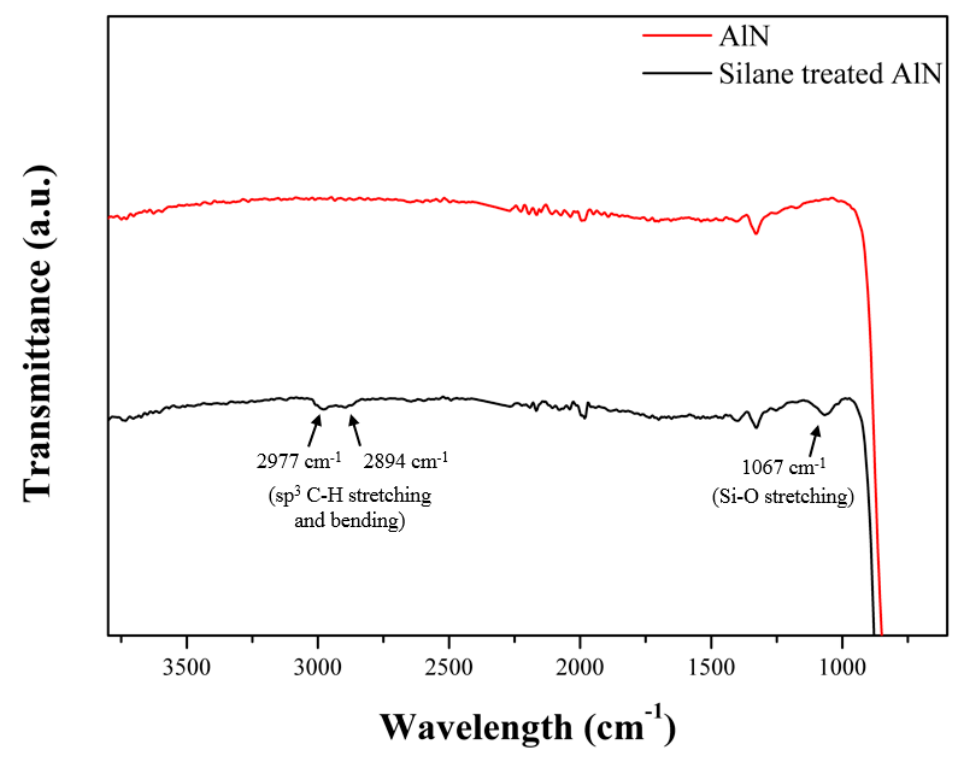

Figure 2. FT-IR spectra of the AlN and silane treated AlN.

Additionally, the bonding structure and quantitative analysis of the silane coupling agent introduced on the surface of the AlN particles were performed by XPS analysis simultaneously. The XPS spectrum and elemental composition results were described in Figure 3, and Table 2. In the case of the untreated AlN particles, no peak appeared in the $\mathrm{Si}_{2 p}$ orbital shown in Figure 3a. On the other hand, in the case of silane treated $\mathrm{AlN}$, the single deconvolution peak appeared at $101.6 \mathrm{eV}$ in the $\mathrm{Si}_{2 \mathrm{p}}$ orbital attributed to the $-\mathrm{Si}-\mathrm{O}-\mathrm{N}$ - bonding shown in Figure $3 \mathrm{~b}$. Moreover, two deconvolution peaks appeared at 396.7 and $399.2 \mathrm{eV}$ in the $\mathrm{N}_{1 \mathrm{~s}}$ orbital attributed to $-\mathrm{N}-\mathrm{Al}-$ bonding and $-\mathrm{N}-\mathrm{O}-\mathrm{Si}-$ bonding, respectively (Figure 3c). It was confirmed as a result of the quantitative analysis for the elements that about 4.6 at \% of the silane coupling agent was introduced on the surface of the AlN particles [28,29]. 
Table 2. Relative percentages of elemental composition of the AlN and silane treated AlN.

\begin{tabular}{cccccc}
\hline \multirow{2}{*}{ Sample } & \multicolumn{5}{c}{ Elemental composition (at \%) } \\
\cline { 2 - 6 } & Al & N & C & O & Si \\
\hline AlN & 30.8 & 15.2 & 28.5 & 25.5 & - \\
$\begin{array}{c}\text { Silane treated } \\
\text { AlN }\end{array}$ & 20.7 & 9.2 & 35.2 & 30.3 & 4.6 \\
\hline
\end{tabular}
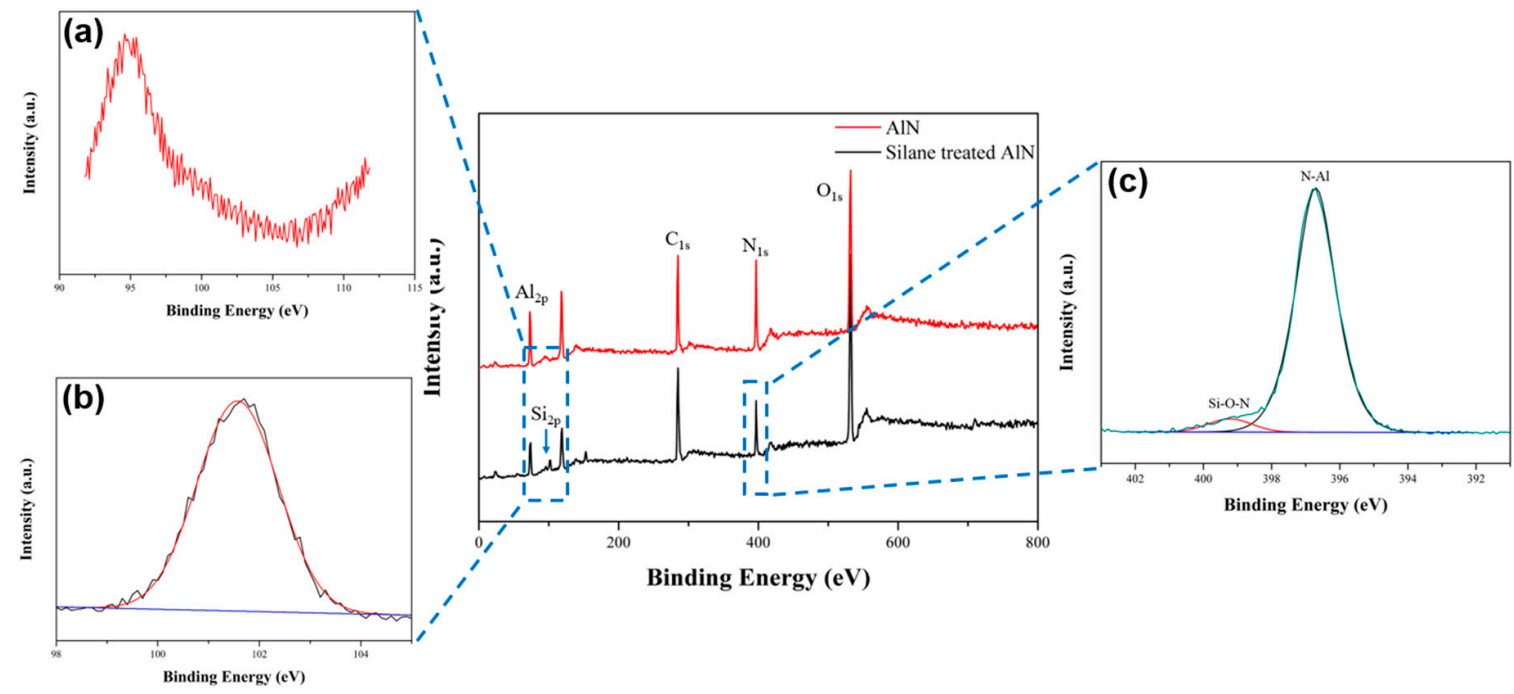

Figure 3. XPS spectra of the AlN and silane treated AlN. (a) $\mathrm{Si}_{2 p}$ peak of the AlN; (b) $\mathrm{Si}_{2 p}$ peak of silane treated AlN; and (c) $\mathrm{N}_{1 \mathrm{~s}}$ peak of the silane treated AlN.

\subsection{Characterization of the Grafting Reaction of AlN Particles on the MWCNTs}

In order to confirm the AlN particle was actually grafted on the MWCNTs, the change in the transmittance with time in ethanol suspension was observed using UV-Vis as shown in Figure 4. In the case of the silane treated AlN, it was precipitated easily in ethanol similar to the untreated AlN. In the case of the AlN-fMWCNTs, the transmission was slightly reduced due to the improved dispersion of MWCNTs by covalent functionalization in ethanol, but did not decrease much because the grafting reaction did not occur. In the case of the AlN grafted MWCNTs, the transmission was drastically reduced compared with that of the AlN-fMWCNTs. This difference originated from the grafting reaction of the AlN on the MWCNTs, it is clearly demonstrated the AlN particle was grafted well on the MWCNTs.

The morphology of the hybrid filler system with the grafting of the AlN particles on the MWCNTs was observed by FE-SEM as shown in Figure 5. Figure 5a,b were the FE-SEM images of the untreated AlN particles and the AlN grafted MWCNTs, respectively. It was confirmed that the grafting reaction of the AlN on the MWCNTs was performed well by observing the attached AlN particles on the surface of the MWCNTs.

Additionally, the morphology of the hybrid filler system was observed by TEM as shown in Figure 6. At this time, Figure 6a,b were TEM images of the fMWCNTs and the AlN grafted MWCNTs, respectively. For the morphology of the fMWCNTs, little defects which were formed by introducing the functional groups, were observed on the surface of the MWCNTs. On the other hand, for the morphology of the AlN grafted MWCNTs, the AlN particles having $4 \mu \mathrm{m}$ particle size were observed on the surface of the MWCNTs. It was further confirmed that the AlN particle was grafted well on the MWCNTs. 


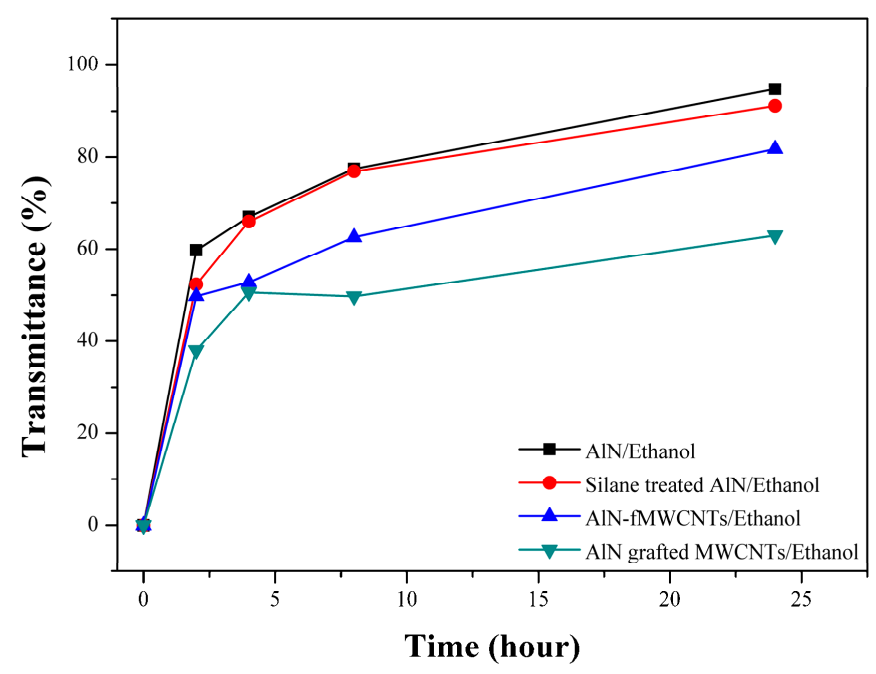

Figure 4. UV-Visible transmittances of the MWCNTs/AIN in ethanol depending on the time.
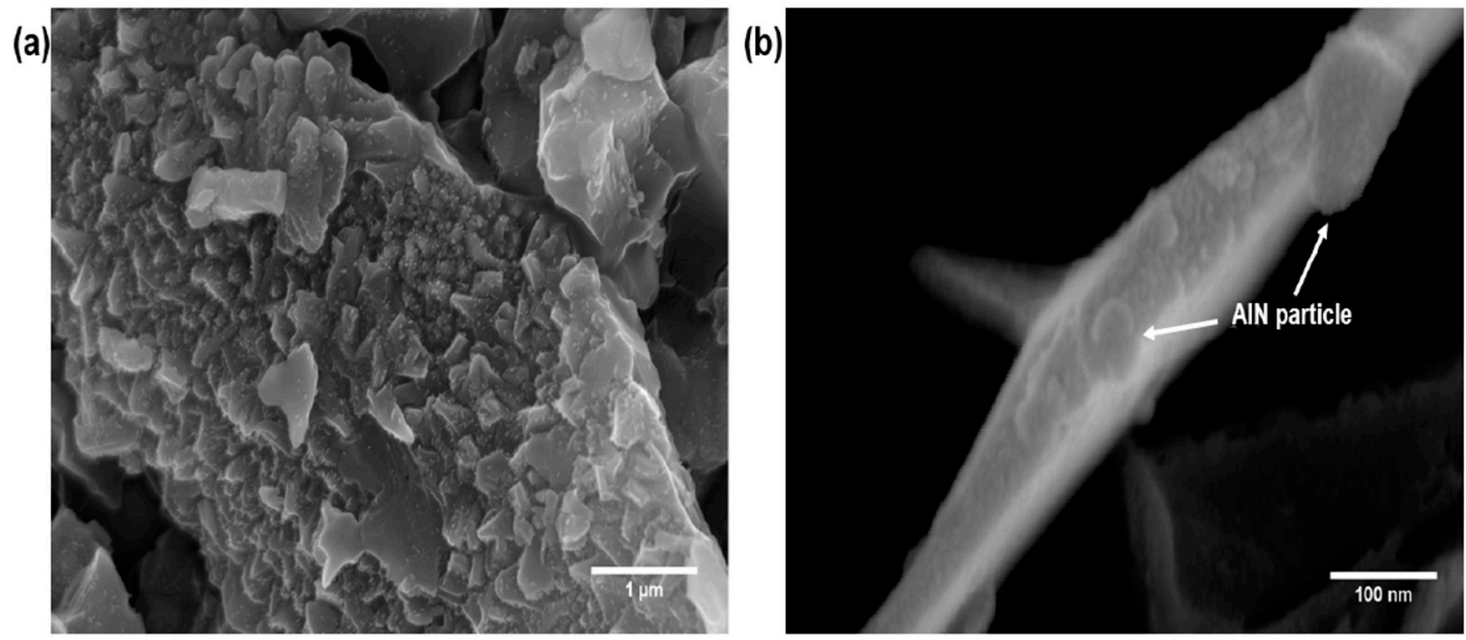

Figure 5. FE-SEM image of (a) AlN particles; and (b) AlN grafted MWCNTs.

(a)

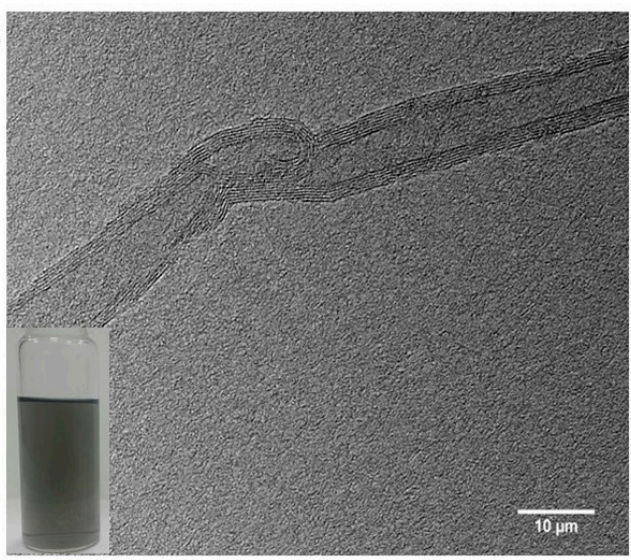

(b)

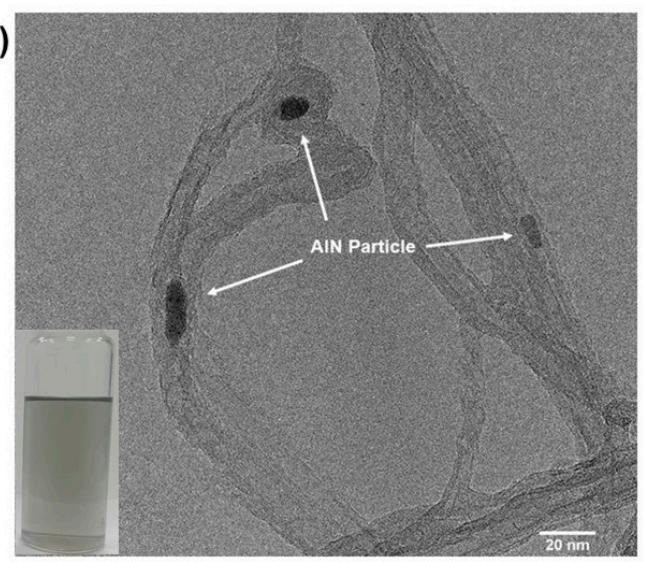

Figure 6. TEM image of (a) fMWCNTs; and (b) AlN grafted MWCNTs (Inserted images were the MWCNTs dispersion in Ethanol medium). 


\subsection{Thermal Properties of the PPS/MWCNTs/AlN Composites}

The thermal conductivity of the polymeric materials emerges by propagating the lattice vibrations generated by interatomic interactions of the phonons within the material $[30,31]$. However, the phonon propagating through the material is scattered due to the thermal resistance, which comes from phonon-phonon scattering, boundary scattering, and defect or impurity scattering. Therefore, it is most important to minimize the phonon scattering to improve the thermal conductivity [30,32]. To propagate the phonons easily within the material, the heat transfer path is very important. When the filler is introduced in a polymer matrix, its density, shape, particle size, and aspect ratio are the factors affecting the formation of the heat transfer path $[30,32,33]$. As mentioned in the introduction, the grafting reaction between the fillers was chosen as one way to form the heat transfer path $[21,22]$. The thermal conductivity of the PPS/MWCNTs/AlN composite depending on the grafting reaction was compared in Figure 7. The thermal conductivity of the PPS/MWCNTs/AlN composite tends to increase in proportion to the AlN contents from the PPSHP1 reported regardless of the grafting on the MWCNTs compared with the neat PPS $(0.28 \mathrm{~W} / \mathrm{mK})$ and PPSHP1 $(0.85 \mathrm{~W} / \mathrm{mK})$ [34]. Also, It was originated from AlN grafted MWCNTs was more uniformly dispersed than not grafted on the PPS matrix by observing a higher thermal conductivity value for PPSHP1sAlN than PPSHP1AlN. It was confirmed the AlN grafted MWCNTs acts as a heat transfer path contributed to promote the phonon propagation, which results in a higher thermal conductivity value [35].

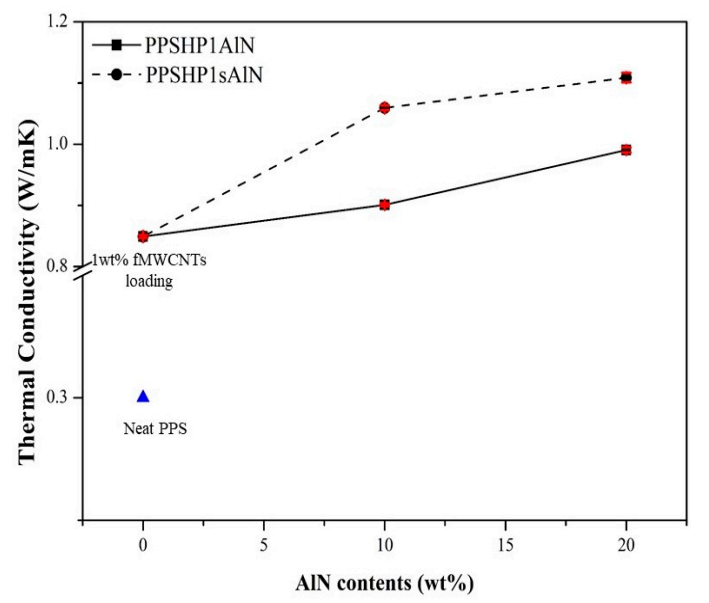

Figure 7. Thermal conductivity of the PPS/MWCNTs/AlN composites.

Figure 8 shows the TGA thermogram of the PPS/MWCNTs/AlN composite. The TGA characteristic thermal data for the PPS/MWCNTs/AIN composites were shown in Table 3. The heat-resistance index was the value used to evaluate the thermal resistance of the polymer composites. The heat-resistance index was calculated using Equation (1) with the decomposition temperature $\left(T_{5}\right.$ and $T_{30}$ ) from the $5 \%$ and $30 \%$ weight losses, respectively [36].

$$
T_{\text {heat-resistance index }}=0.49 \times\left[T_{5}+0.6 \times\left(T_{30}-T_{5}\right)\right]
$$

When the fMWCNTs was introduced on the PPS matrix, the heat-resistance index was slightly improved from $252.90{ }^{\circ} \mathrm{C}$ for neat PPS to $255.87{ }^{\circ} \mathrm{C}$ for PPSHP1. Moreover, it was confirmed that the addition of AlN particles to PPSHP1 highly increased the thermal stability in proportion to the AlN contents due to the intrinsic thermal properties of the AlN regardless of the grafting on the MWCNTs. Interestingly, in case of the PPSHP1sAIN, since the bond between the functional group of the fMWCNTs and the GPTMS introduced on the surface of AlN particles was decomposed between 400 and $500{ }^{\circ} \mathrm{C}$, the heat-resistance index was lower than that of the PPSHP1AIN [37]. 


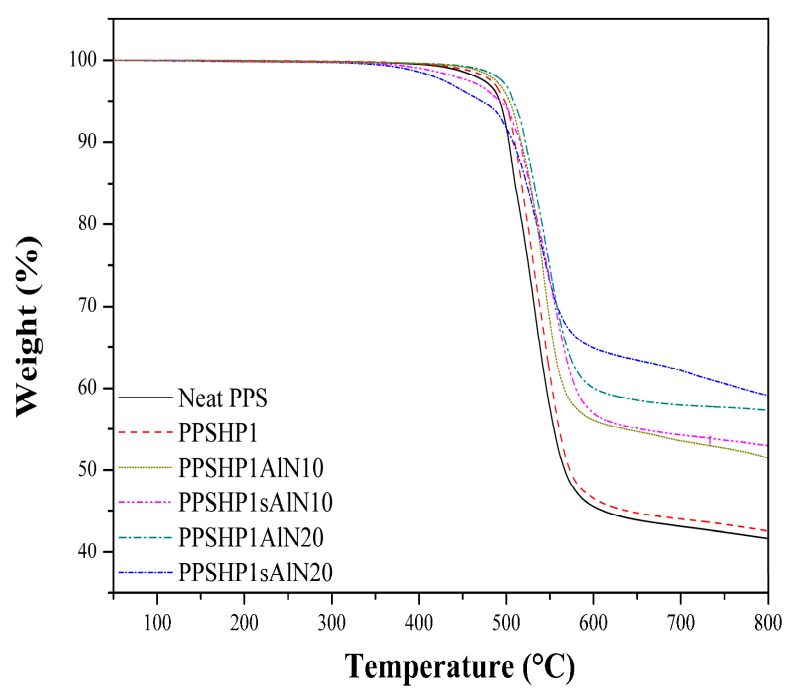

Figure 8. TGA thermogram of the PPS/MWCNTs/AlN composites.

Table 3. TGA characteristic thermal data of the PPS/MWCNTs/AIN composites.

\begin{tabular}{cccc}
\hline \multirow{2}{*}{ Sample } & \multicolumn{2}{c}{ Temperature at weight loss $\left({ }^{\circ} \mathbf{C}\right)$} & Heat-resistance index $\left({ }^{\circ} \mathbf{C}\right)$ \\
\cline { 2 - 3 } & $\mathbf{5} \mathbf{~ w t} \%$ & $\mathbf{3 0 ~} \mathbf{~ w t} \%$ & \\
\hline Neat PPS & 492.36 & 531.97 & 252.90 \\
PPSHP1 & 498.12 & 538.23 & 255.87 \\
PPSHP1AlN10 & 503.53 & 547.58 & 259.68 \\
PPSHP1AlN20 & 507.97 & 558.12 & 263.65 \\
PPSHP1sAlN10 & 492.84 & 555.99 & 260.06 \\
PPSHP1sAlN20 & 472.01 & 558.40 & 256.68 \\
\hline
\end{tabular}

Figure 9 shows the DSC melting and cooling curves of the PPS/MWCNTs/AlN composite. Likewise, the DSC characteristic thermal data of the PPS/MWCNTs/AlN composite was shown in Table 4. When the fMWCNTs was introduced on the PPS matrix, the glass temperature $\left(T_{g}\right)$, crystallization temperature $\left(T_{c}\right)$, melting temperature $\left(T_{m}\right)$, and crystallinity $\left(\chi_{c}\right)$ were improved due to their outstanding intrinsic thermal properties. The crystallinity was calculated using Equation (2).

$$
\chi_{c}=\frac{\Delta H_{f}}{w \times \Delta H_{f}^{0}}
$$

where $w, \Delta H_{f}$, and $\Delta H_{f}^{0}$ represent the weight percentage of the PPS, the heat of fusion at the melting point of the PPS/MWCNTs/AIN composites, and the heat of fusion of the crystalline PPS, which was known as $76.5 \mathrm{~J} / \mathrm{g}$ [38], respectively. Many studies have reported that the MWCNTs, which is a nucleation agent, can facilitate the crystallization by the nucleation effect $[11,34,39,40]$. The experimental data were consistent with the literatures on the nucleation effect of the MWCNTs. Likewise, the addition of the AlN particles to the PPSHP1 increased the low $T_{g}$ of the neat PPS, which appeared near $85^{\circ} \mathrm{C}$ and limited to various applications, was amazingly improved by up to $20{ }^{\circ} \mathrm{C}$. Moreover, $T_{c}, T_{m}$, and $\chi_{c}$ were also increased in proportion to the AlN contents due to the intrinsic thermal properties, and nucleation effects of the AlN regardless of the grafting reaction on the MWCNTs. Interestingly, in the case of the PPSHP1sAlN, the $T_{c}$ and $T_{m}$ were not significantly different from the PPSHP1AIN, but the $\chi_{c}$ was further increased by the improvement of dispersion of the AlN particles on the PPS matrix by grafting on the MWCNTs [41]. 

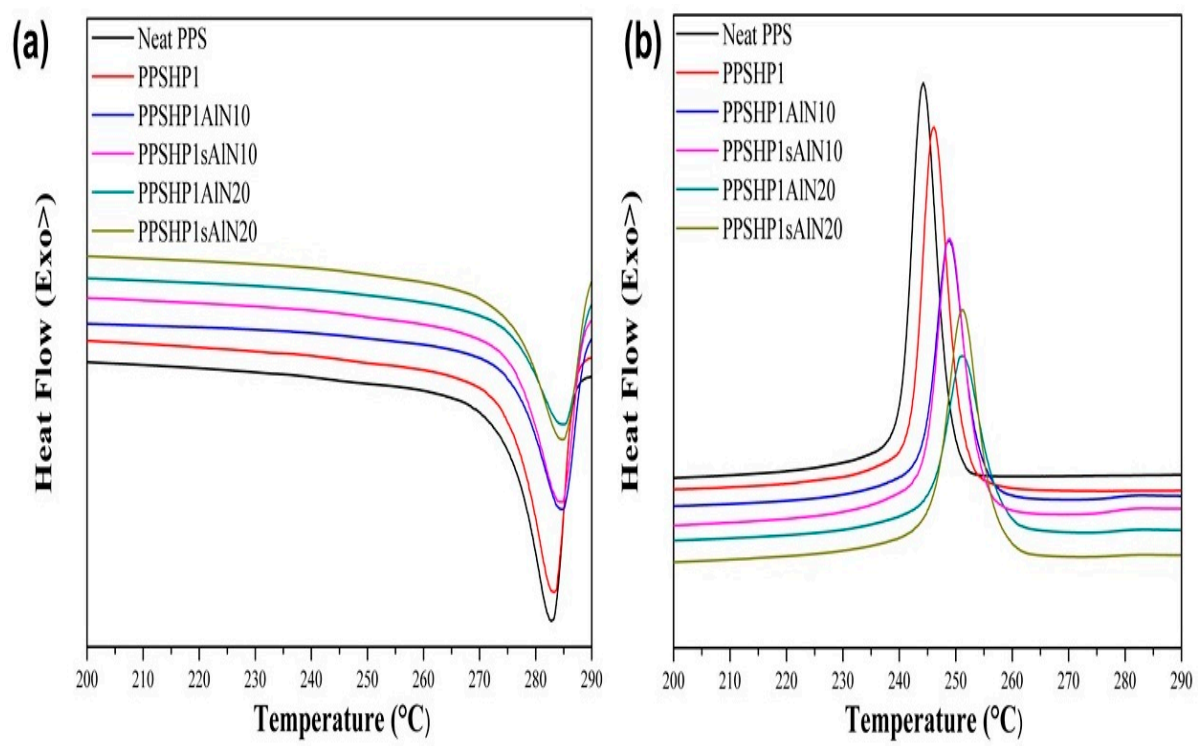

Figure 9. DSC (a) melting curve and (b) cooling curve for $10^{\circ} \mathrm{C} / \mathrm{min}$ heating rate of the PPS/ MWCNTs/AlN composites.

Table 4. DSC characteristic thermal data of the PPS/MWCNTs/AlN composites.

\begin{tabular}{cccccccc}
\hline Sample & $\mathrm{T}_{\mathbf{g}}\left({ }^{\circ} \mathbf{C}\right)$ & $\mathrm{T}_{\mathbf{c}}\left({ }^{\circ} \mathbf{C}\right)$ & $\Delta \boldsymbol{H}_{\boldsymbol{c}}(\mathbf{J} / \mathbf{g})$ & $\mathrm{T}_{\mathbf{m}}\left({ }^{\circ} \mathbf{C}\right)$ & $\Delta \boldsymbol{H}_{f}(\mathbf{J} / \mathbf{g})$ & $\chi_{\boldsymbol{c}}(\%)$ & Ref \\
\hline Neat PPS & 89.09 & 244.28 & 45.97 & 282.84 & 37.06 & 48.44 & \\
PPSHP1 & 94.72 & 246.18 & 45.22 & 283.31 & 40.81 & 53.89 & {$[34]$} \\
\hline PPSHP1AlN10 & 100.75 & 248.77 & 37.69 & 284.65 & 34.21 & 50.25 & \\
PPSHP1AlN20 & 99.71 & 251.13 & 32.40 & 285.04 & 30.69 & 50.78 & - \\
PPSHP1sAlN10 & 102.40 & 248.97 & 39.16 & 284.52 & 35.56 & 52.23 & \\
PPSHP1sAlN20 & 95.29 & 251.08 & 39.39 & 284.79 & 37.03 & 61.27 & \\
\hline
\end{tabular}

\subsection{Morphological Differences of the PPS/MWCNTs/AlN Composites}

The fracture surface was observed by FE-SEM, as shown in Figure 10, to confirm the difference of the dispersion of the hybrid filler on the PPS matrix depending on the grafting of the AlN particle on the MWCNTs. As shown in Figure 10a, the AlN particle was easily aggregated in spherical shape by the interaction between each particle. In the case of the fractured surface of the PPSHP1AlN20, the spherical agglomerated form of the AlN particles was observed [24]. On the other hand, the fractured surface of the PPSHP1sAIN20 was shown in Figure 10b, it was confirmed the homogeneous distribution of the AlN particle was obtained by grafting on the MWCNTs without any agglomerated form between the AlN particles. Therefore, a uniform distribution of the hybrid filler, acting as a heat transfer path allows the phonon to move easily within the material, results in a high thermal conductivity.

Based on the results, the actual distribution of the AlN particles and MWCNTs dependent on the grafting reaction was schematized as shown in Figure 11. In the case of the PPSHP1 shown in Figure 11a, the dispersion of the MWCNTs on the PPS matrix was improved by introduced functional groups introduced by covalent functionalization. In the case of the PPSHP1AlN, as shown in Figure 11b, the agglomerated form of the AlN particles, formed by the interaction between each particle, was represented in Figure 10a, while in the case of the PPSHP1sAlN, since the hybrid filler was distributed uniformly on the PPS matrix as seen in the Figure 10b, it can be schematized as shown in Figure 11c. 
(a)

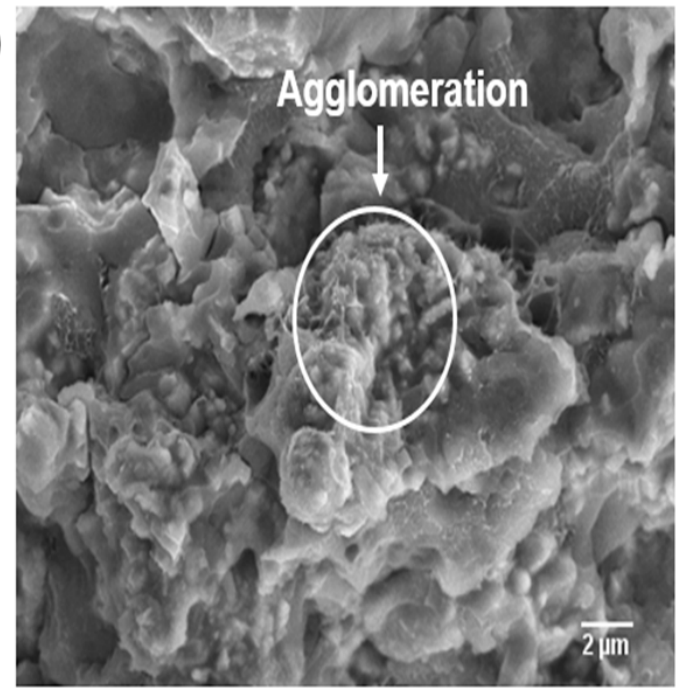

(b)

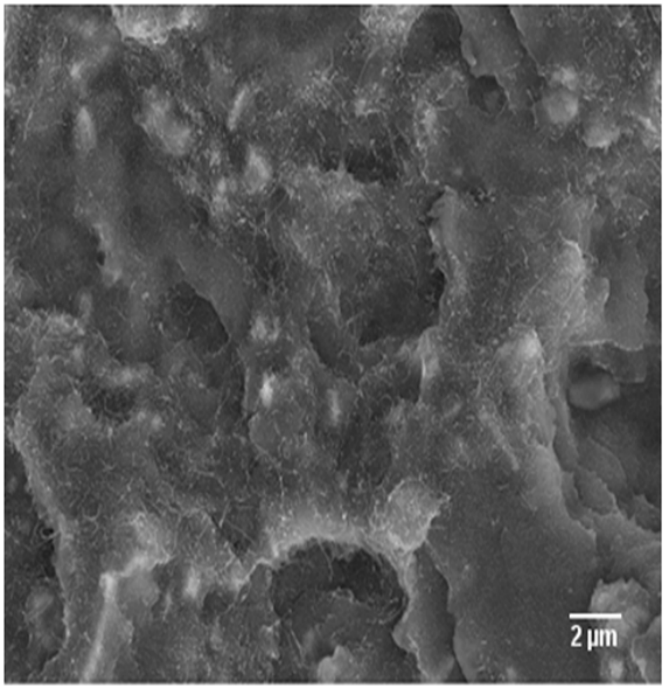

Figure 10. FE-SEM images of the (a) PPSHP1AlN20; and (b) PPSHP1sAlN20.

(a)

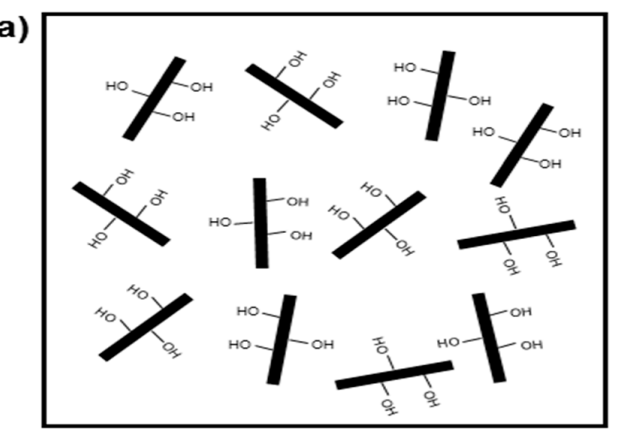

(b)

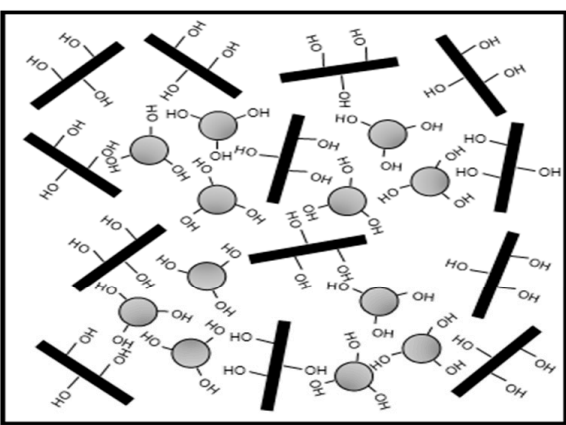

(c)

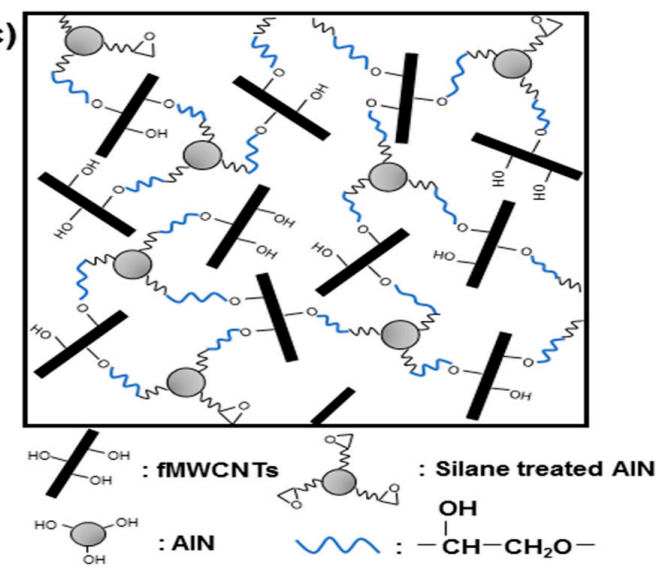

Figure 11. Schemes of the dispersion of the hybrid filler on the PPS matrix. 


\section{Conclusions}

In this study, the PPS composite containing the hybrid filler system was prepared via melt-blending techniques. The hybrid filler systems were divided AlN grafted MWCNTs, and not grafted MWCNTs. To be capable of the grafting reaction with the fMWCNTs, the GPTMS was introduced on the surface of the AlN particles, and confirmed by observing new peaks attributed to the GPTMS in FT-IR and new deconvolution peaks of $\mathrm{Si}_{2 p}$ and $\mathrm{N}_{1 \mathrm{~s}}$ orbitals in XPS analysis. The grafting reaction of the AlN particles on the MWCNTs was also confirmed by observing the transmittance change of the hybrid filler system in UV-Vis, and the morphological change of the MWCNTs in FE-SEM and FE-TEM images. The AlN particle was homogeneously distributed on the PPS matrix in the case of the PPSHP1sAlN. This homogeneous distribution of the hybrid filler, acting as a heat transfer path, contributed to improve the thermal conductivity and thermal resistance compared with those of the PPSHP1AlN. Moreover, the glass transition temperature, which is a limitation of the PPS for application in various industries was significantly improved. The melting temperature and the crystallization temperature were also improved compared with those of the PPSHP1AlN.

Acknowledgments: This research was supported by the "Development of High Stiffness and Thermal Resistant Materials for High Performance Automotive Lighting System" funded by KOPRA Co., Ltd. and the Fundamental R\&D Program for Technology of Materials \& Components (No. 10050526) funded by the Ministry of Trade, Industry and Energy of the Republic of Korea and RIAM: Research Institute of Advanced Materials at Seoul National University.

Author Contributions: Myounguk Kim and Jongshin Park conceived and designed the experiments. Myounguk Kim performed the experiments and wrote the paper. Myounguk Kim and Jongshin Park analyzed the data and Sunmin Park helped with the data analysis and revision of the paper.

Conflicts of Interest: The authors declare no conflict of interest.

\section{References}

1. Rahate, A.S.; Nemade, K.R.; Waghuley, S.A. Polyphenylene sulfide (PPS): State of the art and applications. Rev. Chem. Eng. 2013, 29, 471-489. [CrossRef]

2. Chen, Z.; Li, T.; Yang, Y.; Liu, X.; Lv, R. Mechanical and tribological properties of PA/PPS blends. Wear 2004, 257, 696-707. [CrossRef]

3. Iijima, S. Helical microtubules of graphitic carbon. Nature 1991, 354, 56-58. [CrossRef]

4. Moniruzzaman, M.; Winey, K.I. Polymer Nanocomposites Containing Carbon Nanotubes. Macromolecules 2006, 39, 5194-5205. [CrossRef]

5. McEuen, P.L.; Bockrath, M.; Cobden, D.H.; Yoon, T.G.; Louie, S.G. Disorder, pseudospins, and backscattering in carbon nanotubes. Phys. Rev. Lett. 1999, 83, 5098-5101. [CrossRef]

6. Uchida, T.; Kumar, S. Single wall carbon nanotube dispersion and exfoliation in polymers. J. Appl. Polym. Sci. 2005, 98, 985-989. [CrossRef]

7. Sumita, M.; Sakata, K.; Asai, S.; Miyasaka, K.; Nakagawa, H. Dispersion of fillers and the electrical conductivity of polymer blends filled with carbon black. Polym. Bull. 1991, 25, 265-271. [CrossRef]

8. Yoon, Y.S.; Oh, M.H.; Kim, A.Y.; Kim, N. The Development of Thermal Conductive Polymer Composties for Heat Sink. J. Chem. Chem. Eng. 2012, 6, 515-519. [CrossRef]

9. Kostagiannakopoulou, C.; Fiamegkou, E.; Sotiriadis, G.; Kostopoulos, V. Thermal Conductivity of Carbon Nanoreinforced Epoxy Composites. J. Nanomater. 2016, 2016, 1847325. [CrossRef]

10. Ciecierska, E.; Boczkowska, A.; Kurzydlowski, K.J.; Rosca, I.D.; Hoa, S.V. The effect of carbon nanotubes on epoxy matrix nanocomposites. J. Therm. Anal. Calorim. 2013, 111, 1019-1024. [CrossRef]

11. Deng, S.; Lin, Z.; Xu, B.; Lin, H.; Du, C. Effects of Carbon Fillers on Crystallization Properties and Thermal Conductivity of Poly(phenylene sulfide). Polym. Plast. Technol. Eng. 2015, 54, 1017-1024. [CrossRef]

12. Kim, S.; Kim, T.; Kim, Y.; Choi, H.; Lim, H.; Yang, S.; Park, C. Surface modification for the effective dispersion of carbon nanotubes in solvents and polymer. Carbon 2012, 50, 3-33. [CrossRef]

13. Goyal, R.K.; Jadhav, P.; Tiwari, A.N. Preparation and Properties of New Polyphenylene Sulfide/AlN Composites for Electronic Packaging. J. Electron. Mater. 2011, 40, 1377-1383. [CrossRef] 
14. Mi, Y.; Liang, G.; Gu, A.; Zhao, F.; Yuan, L. Thermally Conductive Aluminum Nitride-Multiwalled Carbon Nanotube/Cyanate Ester Composites with High Flame Retardance and Low Dielectric Loss. Ind. Eng. Chem. Res. 2013, 52, 3342-3353. [CrossRef]

15. Pak, S.; Kim, H.; Kim, S.; Youn, J. Synergistic improvement of thermal conductivity of thermoplastic composites with mixed boron nitride and multi-walled carbon nanotube fillers. Carbon 2012, 50, 4830-4838. [CrossRef]

16. Teng, C.; Ma, C.M.; Chiou, K.; Lee, T. Synergic effect of thermal conductive properties of epoxy composties containing functionalized multi-walled carbon nanotubes and aluminum nitride. Compos. B Eng. 2012, 43, 265-271. [CrossRef]

17. Balázsi, C.; Fényi, B.; Hegman, N.; Kövér, Z.; Wéber, F.; Vértesy, Z.; Kónya, Z.; Kiricsi, I.; Biró, L.P.; Arató, P. Development of $\mathrm{CNT} / \mathrm{Si}_{3} \mathrm{~N}_{4}$ composites with improved mechanical and electrical properties. Compos. B Eng. 2006, 37, 418-424. [CrossRef]

18. Im, H.; Kim, J. Effect of homogeneous $\mathrm{Al}(\mathrm{OH})_{3}$ covered MWCNT addition on the thermal conductivity of $\mathrm{Al}_{2} \mathrm{O}_{3}$ /epoxy-terminated poly(dimethylsiloxane) composites. J. Mater. Sci. 2012, 47, 6025-6033. [CrossRef]

19. Sim, L.C.; Ramanan, S.R.; Ismail, H.; Seetharamu, K.; Goh, T. Thermal characterization of $\mathrm{Al}_{2} \mathrm{O}_{3}$ and $\mathrm{ZnO}$ reinforced silicone rubber as thermal pads for heat dissipation purposes. Thermochim. Acta 2005, 430, 155-165. [CrossRef]

20. Rath, B.N.; Ghanwat, S.J.; Danani, C.; Kulkarni, R.V.; Alur, V.D.; Sathiyamoorthy, D.; Anantharaman, S. Thermal Conductivity of Composites of Beryllia and Lithium Titanate. J. Mater. Eng. Perform. 2013, 22, 3455-3460. [CrossRef]

21. Naito, K.; Yang, J.; Xu, Y.; Kagawa, Y. Enhancing the thermal conductivity of polyacrylontrile- and pitch-based carbon fibers by grafting carbon nanotubes on them. Carbon 2010, 48, 1849-1857. [CrossRef]

22. Zhao, J.; Du, F.; Zhou, X.; Chui, W.; Wang, X.; Zhou, H.; Xie, X.; Mai, Y. Thermal conductive and electrical properties of polyurethane/hyperbranched poly(urea-urethane)-grafted multi-walled carbon nanotube composites. Compos. B Eng. 2011, 42, 2111-2116. [CrossRef]

23. Chung, D.D.L. Materials for thermal conduction. Appl. Therm. Eng. 2001, 21, 1593-1605. [CrossRef]

24. Droval, G.; Feller, J.F.; Salagnac, P.; Glouannec, P. Thermal conductivity enhancement of electrically insulating syndiotactic poly(styrene) matrix for diphasic conductive polymer composites. Polym. Adv. Technol. 2006, 17, 732-745. [CrossRef]

25. Thostenson, E.T.; Ren, Z.; Chou, T.W. Advances in the science and technology of carbon nanotubes and their composites: A review. Compos. Sci. Technol. 2001, 61, 1899-1912. [CrossRef]

26. Kim, M.; Lee, J.; Roh, H.; Kang, H.; Park, S.; Oh, S.; Lee, J.; Park, J. Covalent functionalization of multi-walled carbon nanotubes surface via chemical treatment. J. Nanosci. Nanotechnol. 2017, 17, 2463-2470. [CrossRef]

27. Zhou, Y.; Hu, J.; Chen, X.; Yu, F.; He, J. Thermoplastic Polypropylene/Aluminum Nitride Nanocomposites with Enhanced Thermal Conductivity and Low Dielectric Loss. IEEE Trans. Dielectr. Electr. Insul. 2016, 23, 2768-2776. [CrossRef]

28. Hagio, T.; Takase, A.; Umebayashi, S. X-ray photoelectron spectroscopic studies of $\beta$-sialons. J. Mater. Sci. Lett. 1992, 11, 878-880. [CrossRef]

29. Castanho, S.M.; Moreno, R.; Fierro, J.L.G. Influence of process conditions on the surface oxidation of silicon nitride green compacts. J. Mater. Sci. 1997, 32, 157-162. [CrossRef]

30. Xu, Y.; Chung, D.D.L.; Mroz, C. Thermally conducting aluminum nitride polymer-matrix composites. Compos. A Appl. Sci. Manuf. 2001, 32, 1749-1757. [CrossRef]

31. Zhou, W.; Qi, S.; Li, H.; Shao, S. Study on insulating thermal conductive BN/HDPE composites. Thermochim. Acta 2007, 452, 36-42. [CrossRef]

32. Dashora, P.; Gupta, G. On the temperature dependence of the thermal conductivity of linear amorphous polymers. Polymer 1996, 37, 231-234. [CrossRef]

33. Lee, G.; Park, M.; Kim, J.; Lee, J.; Yoon, H. Enhanced thermal conductivity of polymer composites filled with hybrid filler. Compos. A Appl. Sci. Manuf. 2006, 37, 727-734. [CrossRef]

34. Kim, M.; Lee, J.; Roh, H.; Kim, D.; Byeon, J.; Jee, M.; Park, J. Effects of covalent functionalization of the MWCNTs on thermal properties and non-isothermal crystallization behaviors of the PPS composites. Polymers 2017. accepted. 
35. Choi, S.; Im, H.; Kim, J. The thermal conductivity of embedded nano-aluminum nitride-doped multi-walled carbon nanotubes in epoxy composites containing micro-aluminum nitride particles. Nanotechnology 2012, 23, 65303. [CrossRef] [PubMed]

36. Gu, J.; Du, J.; Dang, J.; Geng, W.; Hu, S.; Zhang, Q. Thermal conductivities, mechanical and thermal properties of graphite nanoplatelets/polyphenylene sulfide composites. RSC Adv. 2014, 4, 22101-22105. [CrossRef]

37. Zhou, G.; Xu, X.; Wang, S.; He, X.; He, W.; Su, X.; Wong, C.P. Surface grafting of epoxy polymer on CB to improve its dispersion to be filler of resistive ink for PCB. Results Phys. 2017, 7, 1870-1877. [CrossRef]

38. Hay, J.N.; Luck, D.A. The conformation of crystalline poly(phenylene sulphide). Polymer 2001, 42, 8297-8301. [CrossRef]

39. Jana, R.N.; Im, C.; Bhunia, H. Effect of multiwalled Carbon Nanotubes on Crystallization Behavior of Poly( $\varepsilon$-caprolactone)diol. J. Thermoplast. Compos. 2009, 22, 531-546. [CrossRef]

40. Reyes-de Vaaben, S.; Aguilar, A.; Avalos, F.; Ramos-de Valle, L.F. Carbon Nanoparticles as Effective Nucleating Agents for Polypropylene. J. Therm. Anal. Calorim. 2008, 93, 947-952. [CrossRef]

41. Guo, B.; Lin, Q.; Zhao, X.; Zhou, X. Crystallization of polyphenylene sulfide reinforced with aluminum nitride composite: Effects on thermal and mechanical properties of the composites. Iran. Polym. J. 2015, 24, 965-975. [CrossRef]

(C) 2017 by the authors. Licensee MDPI, Basel, Switzerland. This article is an open access article distributed under the terms and conditions of the Creative Commons Attribution (CC BY) license (http://creativecommons.org/licenses/by/4.0/). 